\title{
Effects of Ultraviolet Light on Optical Properties of Colloidal CdS Nanoparticles Embedded in Polyvinyl Alcohol (PVA) Matrix
}

\author{
G. Yu. Rudko', A. O. Kovalchuk', V. I. Fediv', , J. Beyer ${ }^{3}$, W. M. Chen ${ }^{3}$, and I. A. Buyanova ${ }^{3}$ \\ ${ }^{1}$ V. Lashkaryov Institute of Semiconductor Physics of National Academy of Sciences of Ukraine, 45, Pr. Nauky, Kiev \\ 03028, Ukraine \\ ${ }^{2}$ Department of Biophysics and Medical Informatics, Bukovinian State Medical University, 42 Kobylyanska st., 58000 \\ Chernivtsi, Ukraine \\ ${ }^{3}$ Department of Physics, Chemistry and Biology, Linköping University, SE-581 83 Linköping, Sweden
}

CdS nanoparticles have been synthesized in aqueous solution using polyvinyl alcohol (PVA) as a capping reagent. The effects of exposure by ultraviolet (UV) light on optical properties of nanocomposites consisting of colloidal CdS nanoparticles and a polymer PVA matrix were studied by employing photoluminescence (PL) spectroscopy. It is shown that UV-induced changes of the photoluminescence intensity in PVA are caused by creation and healing of non-radiative recombination centers. It is also concluded that in the nanocomposites, the UV-induced changes of the PL intensity are predominantly governed by processes at the NP/PVA interface.

KEYWORDS: CdS Nanoparticles, PVA Matrix, Nanocomposites, Photoluminescence.

\section{INTRODUCTION}

Nanocomposites containing semiconductor nanoparticles (NPs) have attracted increasing interest during past decades in view of their potential applications in various fields such as electronics, optoelectronics, biotechnologies, medicine, etc. ${ }^{1,2}$ Nanocomposites consist of a matrix that serves as a binding and supporting medium for NPs and semiconductor NPs themselves. By choosing different materials for matrices and different semiconductors for NPs, properties of composites can be tuned over a wide range. One of rapidly developing research areas focuses on polymer-based composites produced by cheap colloidal methods such as composite polyvinyl alcohol/CdS nanoparticles. In this material system, Polyvinyl alcohol (PVA) provides a transparent and flexible matrix whereas CdS NPs grant efficient light-emission inherent to this direct bandap II-VI semiconductor. Structural properties, photoluminescence (PL) and optical absorption of these nanocomposites have been extensively studied. ${ }^{3-7}$ However, spectroscopic studies of nanocomposites have up to now been predominantly focused on properties of the NPs and much less attention was paid to the polymer matrix. As

\footnotetext{
*Author to whom correspondence should be addressed.

Email: vfediv@ukr.net

Received: 5 February 2012

Revised/Accepted: 14 February 2012
}

a result, a contribution of the polymer to the overall properties of the composite has been mostly overlooked. This is in spite of the fact that intrinsic changes occurring in the polymers under various external influences, such as aging under exposure to ultraviolet (UV) light, could seriously impact performance of possible future composite-based devices. The purpose of the present work is to acquire a detailed understanding of these UV-induced aging effects in the CdS/PVA nanocomposite based on comprehensive PL studies. To distinguish purely photo-induced processes from any other chemical influence, all experiments were carried out in an inert gas (i.e., helium) atmosphere.

\section{EXPERIMENTAL DETAILS}

\subsection{Synthesis of the CdS/PVA Composite}

Colloidal solutions of CdS NPs were synthesized starting with water solution of PVA. Salts of $\mathrm{CdCl}_{2}$ and $\mathrm{Na}_{2} \mathrm{~S}$ were used as precursors for the NPs growth. Synthesis was carried out under ambient conditions. All chemicals were analytical-grade and were used without any further purification. Molecules of PVA served as capping molecules that restricted sizes of the CdS NPs during the growth. To avoid formation of $\mathrm{Cd}(\mathrm{OH})_{2}$ instead of $\mathrm{CdS}$, concentrations of precursors and $\mathrm{pH}$ values were kept within certain limits. Optimal ranges of these parameters were estimated by analyzing the probabilities of possible chemical reactions in 
the solution as $10^{-4}-10^{-2} \mathrm{~mol} / \mathrm{L}$ for the salts and $\mathrm{pH} 3-5$. To maintain a constant supply of the building material for growing NPs, the concentration of the precursors in the growth solution was kept at a constant level. The obtained colloidal solutions of the CdS NPs were thereafter dried at $300 \mathrm{~K}$ in glass Petri dishes that were kept inside a pressure-tight vessel containing an absorbent. As a result of drying, solid nanocomposite films of CdS/PVA were formed. A typical transmission electron microscope (TEM) image of the formed CdS/PVA nanocomposite is shown in Figure 1. Here, the semiconductor NPs appear as dark spots in a light background of the polymer matrix. In addition, grey regions could be seen that are attributed to some products of the reaction between PVA molecules and free ions.

\subsection{Photoluminescence Measurements}

Photoluminescence of the PVA and the nanocomposites was excited using a Coherent Verdi $2 \mathrm{~W}$ laser operating at $\lambda_{\text {exc }}=266 \mathrm{~nm}$. The choice of the excitation wavelength was dictated by the necessity to excite both the unloaded matrix and the CdS NPs in the composite. The wavelength of $266 \mathrm{~nm}$ is sufficiently high to excite chromophore groups (e.g., carbonyl) in the PVA as well as to ensure the band-to-band optical absorption within the $\mathrm{CdS}$ quantum dot. PL signals were registered by a CCD camera (Princenton Instruments ST-133) assembled with an Acton SpectraPro 2500i monochromator. The temperature of measurements was chosen as $200 \mathrm{~K}$. This temperature is low enough to detect all emission bands excited in the nanocomposite ${ }^{8}$ but is sufficiently high to allow internal photochemical transformations in the polymer. The UVtreatment was conducted in inert helium atmosphere, to avoid participation of any extraneous chemical species in the intrinsic UV-induced reconstruction of the polymer and composite.

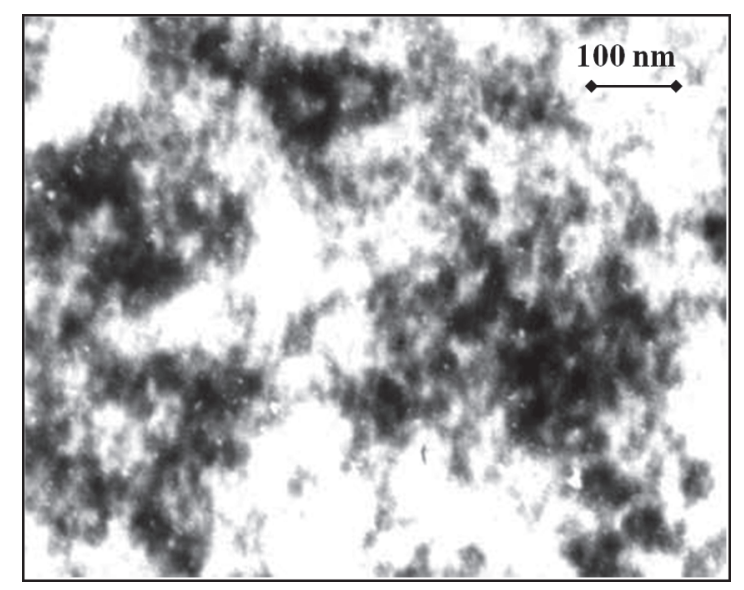

Fig. 1. TEM images of the CdS nanoparticles in the PVA matrix.

\subsection{Procedure of UV Exposure and Kinetics Measurements}

The UV treatment (or aging) of both PVA and nanocomposites of CdS/PVA was carried out at $200 \mathrm{~K}$ (i.e., the same temperature as that in the PL measurements) in helium atmosphere. Three different powers of exciting light $(0.5,2.0$ and $5.0 \mathrm{~mW})$ were used for prolonged illumination and the total time of the UV-exposure at every chosen power was 1 hour. Kinetics of the UV-induced transformations was studied as follows. A PL spectrum of an untreated sample was measured at the very first moment of illumination. To control changes of PL during the course of illumination we measured PL spectra after every 30 seconds of the UV-exposure. The measurements of each PL spectrum lasted for $<0.5$ seconds. Thus, during the total exposure time of 1 hour we obtained 120 spectra that depicted the UV-induced changes in the sample.

\section{RESULTS}

Figures 2(a)-(f) show PL spectra of the unloaded PVA (spectra a-c) and the nanocomposite CdS/PVA (spectra d-f) measured before (red solid curves) and after (black dash curves) the 1-hour-long UV treatment. The PL spectrum of the untreated PVA contains a broad PL band peaking at around $490 \mathrm{~nm}$. This band is usually ascribed in the literature ${ }^{9,10}$ to overlapping emissions from various chromophore groups that were inevitably incorporated in the polymers during the synthesis process. The spectrum of the untreated composite has two broad bands in the green and red spectral ranges. Similar spectra from such composites have been previously reported. ${ }^{11-14}$ Though there is no consensus in the literature as to the exact origin of these bands, most of authors ascribed them to traps within NPs. From Figure 2, the UV treatment is shown to strongly influence the PL spectra of both PVA and nanocomposite leading to considerable changes in both intensities and spectral positions of the detected emissions. For example, for the unloaded PVA a red shift in the PL maximum position is observed after the aging independent of the power of the UV light utilized during the exposure. For the CdS/PVA nanocomposites, instead of two PL bands observed in the untreated samples, only one broad PL band emerges after the treatment at 2 and $5 \mathrm{~mW}$. This band is so broad that it covers the spectral range of both green and red bands that were observed in the PL spectra of these samples before the UV-treatment.

To analyze the influence of the UV treatment in more detail we have studied the kinetics of the UV-induced PL changes. The corresponding results for the unloaded PVA and nanocomposite are shown in Figures 3(a and b), respectively, taking as an example that after the $2 \mathrm{~mW}$ exposure. One notices that in PVA aging causes:

(i) non-monotonous changes in the PL intensity with duration of the exposure; and 


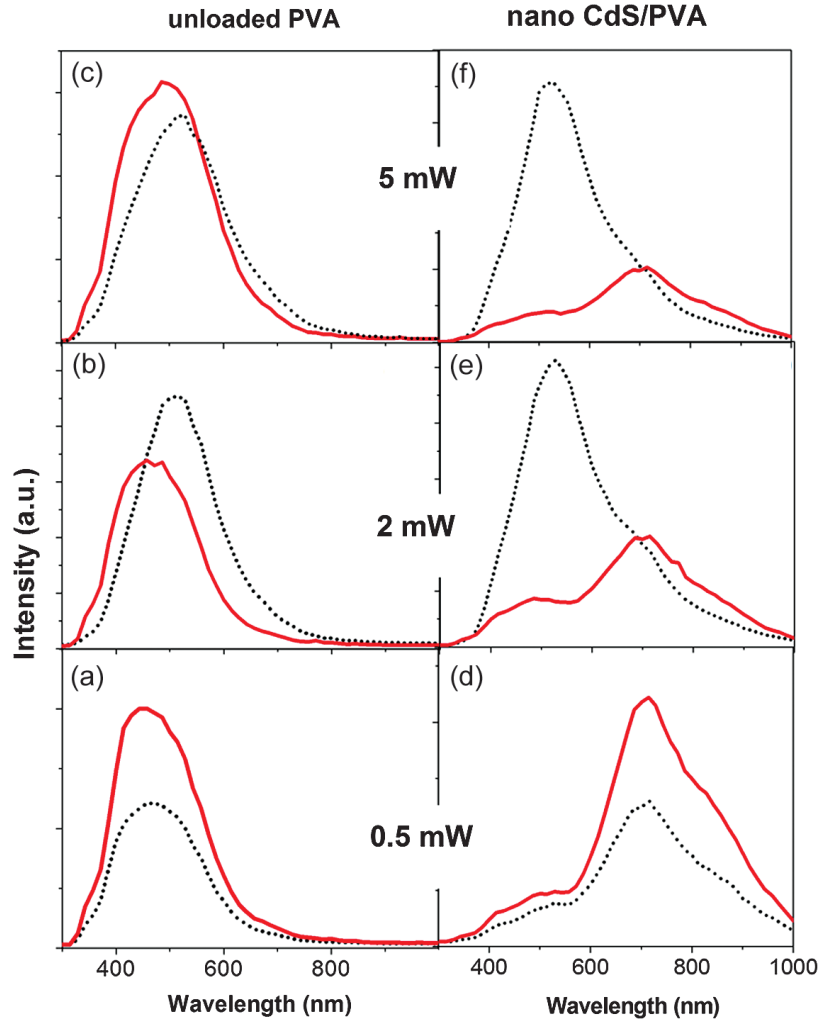

Fig. 2. PL spectra of the unloaded PVA (spectra a-c) and the nanocomposite (spectra d-f) measured before and after the 1-hour UV exposure with excitation powers as specified in the Figure. The spectra obtained before (after) the treatments are shown by red solid curves (black dash curves).

(ii) a red shift of the PL maximum position-see also Figures 4 (a)-(c) where the extracted dependences of the maximum PL intensity (blue solid curves) and the spectral position of PL band (black dash curves) are shown.

Qualitatively similar changes in the PL spectra are also observed for the nanocomposites (see Fig. 3(b) and Figs. 4(d-e)) though the rates of all processes are somewhat different.

Three qualitatively different stages of the UV-induced transformations can be distinguished in the kinetic curves of the PL intensity both for the PVA and for the CdS/PVA. They are most clearly seen in the kinetics of the PVArelated intensity under the $2 \mathrm{~mW}$ excitation-see Figure 4(b) and are labeled as I, II, and III. The stage I is an abrupt and very rapid decrease of the PL intensity. This behavior is more pronounced in pure PVA and at higher UV powers. During the stage II the PL intensity increases. Notably, this increase is characterized by sigmoid-like behavior that is a characteristic of co-operative reactions. The processes at the stage III of the aging are the slowest. Here the PL intensity decreases in the PVA but remains almost constant in the nanocomposites.

It should be noted, however, that based on Figures 4 (a) and (d), the stages II and III are not observed when the treatment was performed with the low excitation

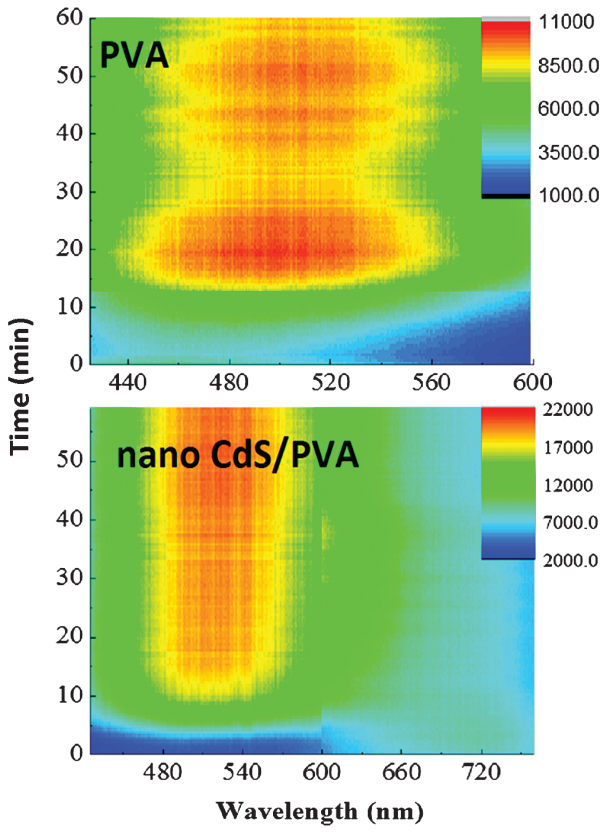

Fig. 3. PL spectra of the unloaded PVA and the nano CdS/PVA nanocomposite measured during exposure with $2 \mathrm{~mW}$ UV light. Relative PL intensities are reflected by the colors, as given in the vertical color bars.

intensity of $0.5 \mathrm{~mW}$. As decreasing light intensity leads to a slow-down of the aging process, we assume that the duration of the UV exposure in our experiments was too short to proceed to stages II and III. Thus, only a drop of the PL intensity is observed during the whole 1-hour-long treatment with weak UV irradiation.

\section{DISCUSSION}

The obtained experimental results evidence that exposure to the UV light strongly influences both the unloaded PVA and the nanocomposite CdS/PVA. Taking into account the known fact that bulk CdS semiconductor is not affected by UV irradiation (with these low light intensities) we conclude that the changes in the nanocomposites are predominantly related to transformations that occur both within the polymer and at interfaces between the polymer and the NPs. Thus, we start our discussion with the analysis of the UV influence on the unloaded PVA.

Polyvinyl alcohol is a polymer with a repeating vinyl alcohol unit that has a molecular formula $\left(\mathrm{C}_{2} \mathrm{H}_{4} \mathrm{O}\right)_{n}$. The UV-induced transformations of the unloaded PVA could be ample and are not fully understood. We can single out several features of PVA that could be affected by exposure to UV light such as

(i) configurational transformations;

(ii) hydrogen bonding between the macromolecules and between neighboring parts of a macromolecule;

(iii) amount and type of chromophore groups. 

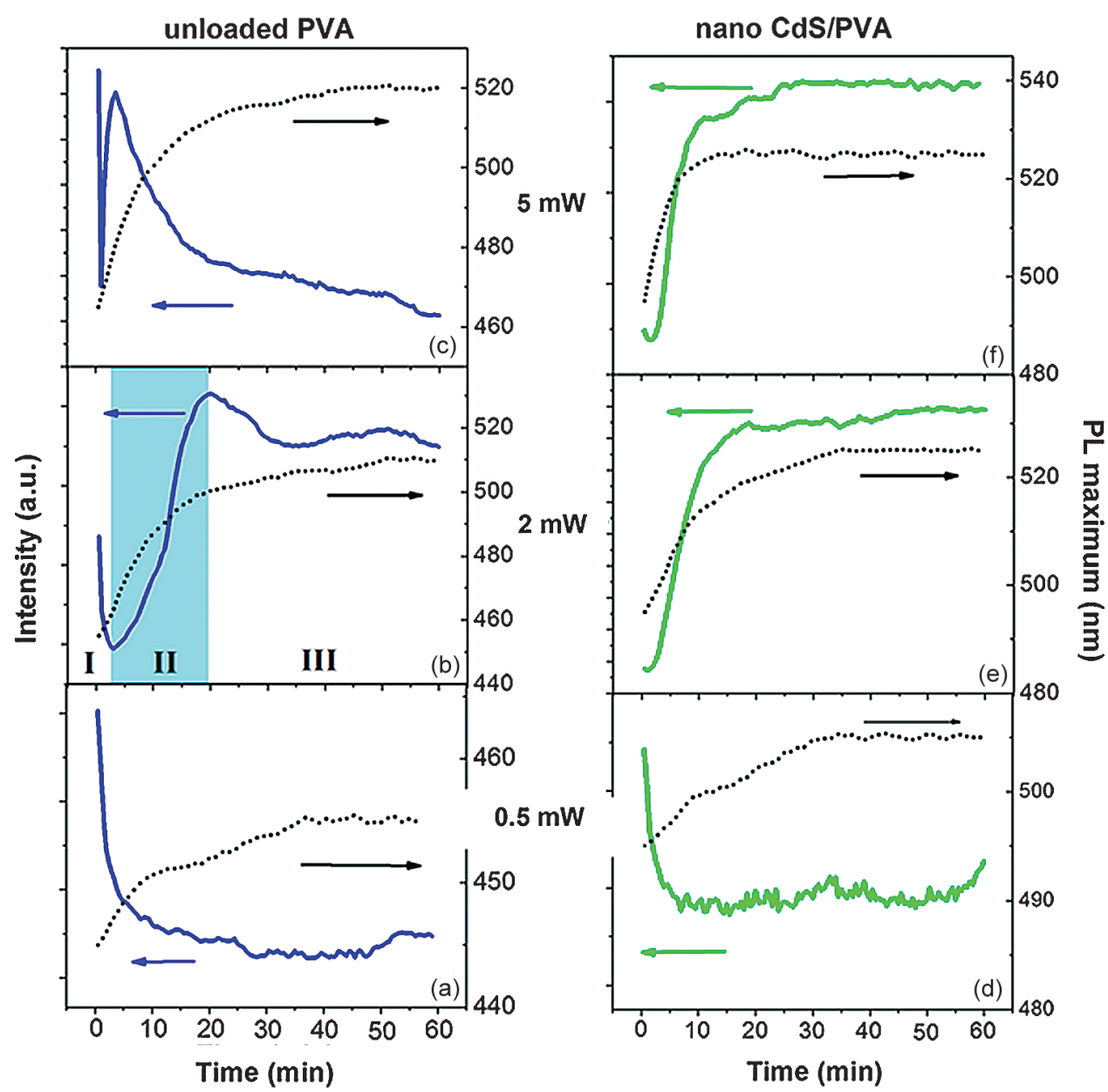

Fig. 4. Spectral positions of the PL maxima (black dash curves) and the PL intensity (blue solid curves for the unloaded PVA and green solid curves for the CdS/PVA nanocomposite) as a function of duration of the UV exposure. (a)-(c) are obtained from the unloaded PVA whereas (d)-(f) correspond to the nano CdS/PVA nanocomposite. The intensities of UV light used for the exposure are as specified. I, II and III in (b) denote the three stages of the material transformation.

(i) It is known that depending on the arrangement of $\mathrm{OH}$ groups along the backbone chain, molecules in pure PVA can exist in forms of isotactic, syndiotactic or atactic conformers. The syndiotactic configuration with its regularly alternating $\mathrm{OH}$ groups on both sides of the polymer chain renders better conditions for regular interchain bonding of macromolecules. These three polymer configurations (conformers) usually coexist. External factors (e.g., UV light) can stimulate mutual transformations of these species that occur via rotation of hydroxyl groups around the chain (these transformations are also named spatial relaxation processes ${ }^{15}$ ). Relative content of different conformers in the polymer backbone chain influences the PL spectra of PVA. The general trend for PL of PVA molecules with different stereoregularities is an increase of the PL wavelength in the sequence syndiotactic-atactic-isotactic configuration. ${ }^{16}$ Thus, the spatial relaxation processes in individual structures of the PVA chain can lead to a shift of the PL spectra. The rotations of hydroxyl groups can be rather quick. For example, Smith et al. ${ }^{15}$ have showed that at the temperatures exceeding $210 \mathrm{~K}$ the relaxation time is about $30 \mathrm{~ms}$.

(ii) One more consequence of high content of hydroxyl groups in the PVA material is that they can take part in hydrogen bonding between the macromolecules. Owing to hydroxyl groups present in each unit of the chain, PVA is characterized by inter- and intra-molecular hydrogen bonding that governs conformational mobility of polymer chains and, thus, influences non-radiative processes rates. ${ }^{17}$

(iii) Another important feature of PVA (which is also a characteristic of other polymers) is the presence of different kinds of radicals and bonds that are not described by the chemical formula of pure PVA. The types and concentrations of imperfections usually depend on the polymer synthesis process. A prevailing majority of commercially available types of PVA are described by the formula $\left(\mathrm{C}_{2} \mathrm{H}_{3} \mathrm{OR}\right)_{n}$ where $\mathrm{R}$ stands for radicals $(\mathrm{R}=\mathrm{H}$ or $\mathrm{COCH}_{3}$ ). Normally many other types of radicals are also present in the material. The substituents are usually placed randomly along the chain and they also contribute 
to atacticity of polymers. The presence of imperfections strongly influences optical properties of PVA-both absorption and emission. Those groups that absorb and emit within the HOMO-LUMO energy gap ${ }^{18,19}$ in the polymer are called chromophore groups. ${ }^{20,21}$ Quite common absorbing and light-emitting species in PVA include carbonyl $(\mathrm{C}=\mathrm{O})$ and carboxyl $(-\mathrm{COOH})$ groups, polyene groups, Lloyd's chromophores with empiric formula $-(\mathrm{CH}=\mathrm{CH})_{n}{ }^{-}$ $\mathrm{CO}-{ }^{22}$ etc. It is also known that under the UV-, $\mathrm{X}-$ and gamma-ray irradiation many new chromophore groups can appear. ${ }^{21,23-28}$ Most important reactions that occur in PVA under UV exposure are reactions of main chain scission, oxidation and loss or conversion of side groups. Given that the UV treatment is carried out in the oxygencontaining atmosphere, the content of carbonyl group increases and the content of hydroxyl group decreases. ${ }^{19,25}$ Another important consequence is an increasing content of polyenes-the groups containing unsaturated $\mathrm{C}=\mathrm{C}$ bonds alternating with saturated $\mathrm{C}-\mathrm{C}$ bonds. Notably, not only the amount of polyenes increases but also the number of unsaturated $\mathrm{C}=\mathrm{C}$ bonds within these chain chromophores increases. ${ }^{19,26}$ Most of these reactions are the result of a rupture of the main chain of PVA under UV influence while cross-linking of macromolecules is more peculiar for $\mathrm{X}$ and gamma-ray irradiation. ${ }^{21,29}$

Several types of processes and counteracting trends should be taken into account while analyzing kinetics of I, II and III stages of the observed UV-induced transformations. The fastest UV-induced process is the enhancement of non-radiative recombination due to deterioration of intraand inter-molecular bonds and conformational transformations of the polymer chain. The origin of this process is as follows. In the steady unperturbed state there is a certain amount of hydrogen bonds between neighboring units of a backbone chain (intra-molecular bonds). There are also ample hydrogen and Wan-der-Waals bonds that are binding macromolecules of polymer to each other (intermolecular bonds) and bonding via intermolecular water. Absorption of the UV photons stimulates breaking of these bonds and rotations of hydroxyl groups around the backbone chain. As a result many bonds are deteriorated and non-radiative recombination of photo-induced carriers is enhanced.

After the destruction of a sufficiently large number of bonds and accumulation of a certain amount of configurational changes in the backbone of the PVA molecules the second UV-induced process is triggered. This process occurs via cooperative reactions of re-shaping of the PVA molecules. Indeed, the breakage of an H-bond in a certain unit of the chain is accompanied by changes of this unit orientation with respect to the backbone. In polymers this rotation is not completely free but correlates with conformational state of neighboring units. As a consequence, restructuring of the $\mathrm{H}$-bonds in the system is a cooperative reaction. ${ }^{30}$ The very first steps of these transformations facilitate the forthcoming ones thus increasing the rate of the reaction until the available possibilities for re-bonding and reshaping start to exhaust. This exhaustion leads to slowing down of the reaction and, finally, to the saturation of the process. The kinetics of such cooperative reactions is described by the characteristic sigmoid-like curves (see Fig. 4(b), stage II).

Two more UV-induced processes are the consequences of the backbone chain rapture. The scission of macromolecular chains leads to both deterioration of the $\mathrm{C}-\mathrm{C}$ bonds and to formation of new chromophores (carbonyls, polyenes, etc.). Respectively, both new non-radiative ${ }^{31}$ and radiative recombination centers appear. These processes have counteracting influence on the PL of PVA and are the slowest among all processes involved in the UV-induced PL changes.

Superposition of all the aforementioned processes determines the shape of kinetic curves that describe the behavior of the PL under the UV exposure. Below we propose a detailed analysis of the contributions of these processes to the PL changes on the every stage of the UV-induced changes.

Kinetics of UV-induced changes in the PL of the unloaded PVA.

Stage I: An abrupt decrease of the PL intensity and increase of the PL wavelength (Fig. Fig. 4(b), stage I).

These changes show that the dominating process at this stage is quick breaking of intra- and inter-molecular bonding, including the Wan-der-Waals and hydrogen bonds as well as bonding via intermolecular water. The breaking facilitates separate acts of the configurational transformations of the PVA chains that change the sterioregularity of $\mathrm{OH}$ groups along the backbone chain. The broken bonds serve as the centers of non-radiative recombination and cause the decrease of PL intensity. Configurational transformations lead to the decrease of a share of syndiotactic and atactic PVA bonding in favor of the isotactic configuration which leads to the observed red shift of the PL maximum.

It should be noted that all aforementioned processes are accompanied by a much slower process-a formation of new chromophore groups in the long polymer molecules via chain scission. At the stage I (and at the stage II as well) this slow process is overwhelmed by other much faster processes. Nevertheless, the formation of the new chromophore groups is the main cause of the PL spectral shift at all stages of the UV-induced transformations.

Stage II: The PL intensity strongly increases and the PL wavelength continues to steadily increase but with a slower rate-Figure 4(b).

The kinetics of PL intensity demonstrates a typical sigmoid-like shape characteristic for cooperative reactions pointing to the cumulative action of all transformations that were produced during the previous stage I. It means that after the destruction of a sufficiently large number of bonds and accumulation of a certain amount of 
the configurational changes in the backbone of the PVA molecules, the cooperative reactions of re-shaping of the PVA molecules are triggered. Re-bonding between the PVA chains eliminates the non-radiative centers that were formed during the stage I and, as a result, the PL intensity increases. The increase of a share of molecules in the isotactic configuration together with emergence of new chromophore groups lead to the increase of the PL wavelength.

Stage III: The PL intensity saturates (at low intensities of UV light) or decreases (at high intensities of UV irradiation) and the wavelength of PL almost saturates.

The reconstruction of the backbone chains via the configurational transformations has finished during the stages I and II, and now the scission of the PVA backbone chains remains the dominating process. As have been stated above, two consequences of this process are the formation of chromophores and the creation of non-radiative centers. The observed saturation of the spectral shift implies that the chromophores species of new types (the ones that emit light at even longer wavelengths) do not emerge. However, the creation of new chromophores of already existing types and non-radiative centers are the counteracting factors that regulate the PL intensity. At $2 \mathrm{~mW}$ irradiation these factors compensate each other while at $5 \mathrm{~mW}$ irradiation the non-radiative recombination dominates.

Kinetics of the UV-induced changes in the PL of the nanocomposite PVA/CdS.

As a preface to the discussion of composite behavior we would like to stress that UV light with the wavelength $266 \mathrm{~nm}$ is weakly absorbed by the PVA and strongly absorbed by the NPs. Thus, the energy of light that illuminates the composite is shared between the NPs and the matrix. Therefore the effective intensity of light that influences the PVA within the composite must be lower than that for the unloaded PVA. As a result one should expect lower rates of the UV-induced transformations in the composite.

Despite of expectations of quantitative distinctions, our experimental results show qualitatively similar kinetics of the UV-induced changes of the PL intensity in the nanocomposite (Figs. 4(d-f)) and in the PVA (Figs. 4(a-c)). Moreover, similar to the PVA, three stages of the UV-induces transformations can also be distinguished in the kinetic curves for the nanocomposite. Thus, one can conclude that all types of the processes that occur under illumination in the unloaded polymer do take place in the nanocomposite as well. This holds, first of all, for the "bulk", unperturbed part of the polymer matrix, i.e., for those regions that are not in contact with the NPs. Different behavior could be expected for the polymer molecules at the interface between the NP and the PVA. Thus, we will concentrate predominately on the distinctive features of the PL changes in the composite that are related to the NPs and the interface.

Stage I: It is seen from Figures 4(d)-(f) that similarly to the unloaded polymer (Figs. $4(\mathrm{a}-\mathrm{c})$ ) the PL intensity for the nanocomposite decreases. However, the drop of the intensity is less pronounced. In the same way behaves the wavelength of the PL maximum: it increases but the shift is smaller than that for pure PVA. It is known that adding salts to polymers deteriorates hydrogen bonding between macromolecules. Judging from the observed behavior of the nanocomposite at the stage I, Figures 4(d)-(f), the number of hydrogen bonds in the nanocomposite is much lower than that in pure PVA. Thus, the decrease of the PL intensity in the nanocomposite is not as pronounced as in the PVA. In the nanocomposites UV light also destroys hydrogen bonds between the surface of the NP and neighboring polymer molecules. Henceforth, the broken bonds at the interface tend to re-structure and initiate the formation of new radiative recombination centers (during stage II).

Stage II: The increase of the PL intensity and the red shift of the PL maximum in stage II occur almost simultaneously. Thus we suppose that both changes are related to the same process-creation of new radiative centers on the surface of the NPs via re-bonding between the NP and neighboring polymer molecules that underwent the conformational transformations. The formation of new radiative centers is supported by drastic changes in the PL spectra (Figs. 2(e, f)): instead of the green and red PL bands only one very broad PL band that covers the spectral range of both emissions emerges. The large width of the PL band implies that a wide variety of interfacial energy levels formed.

Stage III: Stage III is characterized by the saturation of both PL intensity and spectral shift (Figs. 4(e, f)). Thus one can conclude that rebonding is completed during the stage II.

\section{CONCLUSION}

We have shown that the exposure to UV light causes important changes in both PVA and nanocomposite, though the photo-induced transformations in the nanocomposite occur at a lower speed. It is suggested that a possible reason for the observation is partial absorption of the UV light by the NPs, which effectively leads to lower light intensity that influences the matrix in the nanocomposite as compared with that in the unloaded polymer. Two processes are suggested to be responsible for the UV-induced changes of the PL spectra in the PVA:

(i) creation and destruction of non-radiative recombination centers due to changes of an amount of hydrogen bonds as well as conformational changes of the PVA backbone, and (ii) the formation of new light-emitting species (chromophores) in the polymer molecules.

The UV-induced changes of the PL spectra in the nanocomposite are shown to be predominantly governed by the processes at the NP/polymer interface.

These include: 
(i) rupture of the existing bonds between the NP and the surrounding polymer molecules that results in the formation of unbounded surface species that act as non-radiative recombination centers,

(ii) formation of new bonds, which eliminate channels of non-radiative recombination; and

(iii) creation of new light-emitting states at the interface.

Acknowledgments: Financial support from the Swedish Institute via Visby program is greatly appreciated.

\section{References and Notes}

1. P. Reiss, E. Couderc, J. De Girolamo, and A. Pron, Nanoscale 3, 446 (2011).

2. K. M. L. Taylor-Pashow, J. D. Rocca, R. C. Huxford, and W. Lin, Chem. Commun. 46, 5832 (2010)

3. H. S. Mansur, A. A. P. Mansur, and J. C. González, Polymer 52, 1045 (2011).

4. I. S. Elashmawia, N. A. Hakeema, and M. S. Selim, Mater. Chem. Phys. 115, 132 (2009).

5. F. El-Tantawy, K. M. Abdel-Kader, F. Kaneko, and Y. K. Sung, Eur. Polym. J. 40, 415 (2004).

6. J. C. Ferrer, A. Salinas-Castillo, J. L. Alonso, S. Fernandez de Evila, and R. Mallavia, Physics Procedia 2, 335 (2009).

7. P. K. Khanna, R. R. Gokhale, V. V. V. S. Subbarao, N. Singh, K.-W. Jun, and B. K. Das, Mater. Chem. Phys. 94, 454 (2005).

8. V. I. Fediv, G. Yu. Rudko, A. I. Savchuk, E. G. Gule, and A. G. Voloshchuk, Physica E (In print).

9. J. Mieloszyk, R. Drabent, and J. Siódmiak, J. Appl. Polym. Sci. 34, 1577 (1987).

10. W. Chen, G. Huang, H. Lu, D. E. McCready, A. G Joly, and J.-O. Bovin, Nanotechnology 17, 2595 (2006).

11. H. Wang, P. Fang, Z. Chen, and S. Wang, J. Alloy. Compd. 461, 418 (2008).
12. I. Caramana, S. Robu, P. Gaşin I. Lazar, G. Lazar, and M. Stamate, Proceedings of the SPIE 7297, 729717 (2009).

13. M. Pattabi and A. B. Saraswathi, Compos. Interface. 17, 103 (2010).

14. S. Madan, J. Kumar, I. Singh, D. Madhwal, P. K. Bhatnagar, and P. C. Mathur, Phys. Scr. 82, 045702 (2010).

15. K. J. Smit, R. Sakurovs, and K. P. Ghiggino, Eur. Polym. J. 19, 49 (1983).

16. S. Ram and T. K. Mandal, Chem. Phys. 303, 121 (2004).

17. J. M. de Sousa, P. N. M. dos Anjos, E. C. Pereira, Y. G. Gobato, and L. S. dos Santos, Synth. Metals 130, 121 (2002).

18. S. Mahendiaa, A. K. Tomara, and S. Kumar, Mater. Sci. Eng. B 176, 530 (2011).

19. L. Congxu, C. Hongying, and L. Dongyuan, Radiat. Phys. Chem. 42, 229 (1993)

20. M. Abdelaziz and E. M. Abdelrazek, Physica B 390, 1 (2007).

21. H. Kaczmarek and A. Podg'orski, J. Photoch. Photobio. A 191, 209 (2007).

22. D. G. Lloyd, J. Appl. Polym. Sci. 1, 70 (1959).

23. S. P. Vijayalakshmi and G. Madras, J. Appl. Polym. Sci. 101, 233 (2006); J. Appl. Polym. Sci. 100, 4888 (2006); J. Appl. Polym. Sci. 102, 958 (2006).

24. D. He, H. Susanto, and M. Ulbricht, Prog. Polym. Sci. 34, 62 (2009).

25. H. Aoki, M. Uehara, T. Suzuki, and A. Yoshida, Eur. Polym. J. 16, 571 (1980).

26. K. Maruyama, Y. Kuramoto, M. Yagi, and Y. Tanizaki, Polymer 29, 24 (1988).

27. E. Baimuratov, D. S. Saidov, and I. Y. Kalontarov, Polym. Degrad. Stabil. 39, 35 (1993).

28. Y. Chen, Z. Sun, Y. Yang, and Q. Ke, J. Photoch. Photobio. A 142, 85 (2001).

29. S. Akhter, K. Allan, D. Buchanan, J. A. Cook, A. Campion, and J. M. White, Appl. Surf. Sci. 35, 241 (1988)

30. L. V. Pavlova, S. A. Pavlov, and M. A. Bruk, Polymer Science U.S.S.R. 30, 957 (1988).

31. Z. Zainuddin, D. J. T. Hill, and T. T. Le, Radiat. Phys. Chem. 62,283 (2001). 